dass die Steinkohlen des Beckens von Pées durchschnittlich 1,09 Proc., während die Lignitkohlen 20,98 Proc. Feuchtigkeit enthalten, welche bei frisch geförderter Koble bis zu 35 bis 40 Proc. steigen kann. Nehmen wir den durchschnittlichen Wasserstoffgehalt und berechnen, wie viel Wasser bei dessen Verbrennung entsteht, so finden wir bei der Steinkohle 35,01 Proc., bei der Lignitkohle 29,97 Proc. Die Feuergase führen insgesammt bei der Lignitkohle 50,95 Proc., bei der Steinkoble 36,10 Proc. der Kohlen als Wasser ab, es ist daher bei der Lignitkohle ein viel grösserer Wasserüberschuss vorhanden, welcher mit der Schwefligsäure Schwefelsāure bilden kann.

Der durchschnittliche schädliche Schwefelgehalt beträgt bei der Lignitkohle 3,60 Proc., während bei der Steinkohle dieser nur 2,83 Proc. ist. Der calorische Werth ist bei ersterer 3580, bei letzterer 6055 Calorien. Es müssen daher von der Lignitkoble ungefähr 41 Proc. mehr verbrannt werden als von der Steinkohle. Im Betriebe ist die Differenz 50 Proc. In Folge dessen verbrennt bei der Lignitkohle bei gleicher Wärmewirkung beinahe die doppelte Schwefelmenge als bei der Steinkohle, und zwar ist das Verbältniss 2,83:5,07, im Betriebe 2,83:5,40. Unter solchen Umständen müssen die Feuerbüchsen in allerkürzester Zeit ausgewechselt werden.

Bei der Steinkohle ist trotz des kleineren Schwefelgehaltes in einem Falle die zerstörende Wirkung des Schwefels nachgewiesen, und so komme ich wie F. Fischer zu dem Schlusse, dass Koblen mit hohem Schwefelgehalt nur dann schädlich wirken, wenn sie viel Feuchtigkeit enthalten, oder in Folge Undichtigkeit Wasser zugeführt wird. Kohlen, die relativ trockene Verbrennungsproducte liefern, üben trotz des hohen Schwefelgehaltes keinen schädlichen Einfluss auf die Feuerbüchse. Diese Thatsache steht auch damit im Einklange, dass die angeführte Steinkohle, trotz des grossen Schwefelgehaltes, bei den auf der Fiumaner Linie verkehrenden Locomotiven, wo sie schon seit langen Jahren in Verwendung ist, keine sichtbaren Zerstörungen hervorgerufen hatte. Sobald aber Wasser in Folge Undichtigkeit in die Feuerbüchse gelangt, werden nach der Zeit die schädlichen Einflüsse des Schwefels bemerkbar. Wir sind daher bei Kohlen mit relativ trockenen Verbrennungsproducten im Stande, durch sorgfältige Instandhaltung des Kessels den schädlichen Einfluss der Koblen auf das minimale Maass zu beschränken. Bei allen anderen
Kohlen wie diese Lignitkohle stehen wir machtlos da und es bleibt nichts übrig, als solche garnicht zu verwenden.

Es wäre noch eine Frage zu beantworten, wie sich Stahlfeuerbüchsen gegen stark schwefelhaltige Kohlen, wie angeführte Lignitkohle, halten werden. Wenn wir in Betracht ziehen, dass Eisen gegen Säuren viel geringeren Widerstand leistet als Kupfer, so können wir mit Bestimmtheit behaupten, dass Stahlfeuerbüchsen viel kürzere Zeit halten werden als solche von Kupfer.

Laboratorium der K. ung. Staatseisenbahnen.

\section{Über Trockenapparate.}

$$
\text { Von }
$$

\section{Max Kaehler.}

Nach dem von Hugo Petersen gehaltenen Vortrag: "Über Trockenapparate für die chem. Laboratorien" (d. Z. 1892 S. 536) hat ein Trockenapparat folgende Forderungen zu erfüllen:

1. verschiedene constante Temperatur bei genügender Geräumigkeit, 2. starke Ventilation durch den Trockenraum zur Fortführung des verdampfenden Wassers, ohne Schädigung der gleichmässigen Temperatur des Trockenraumes und 3. Zuführung der Wärme durch directe Leitung von der Heizfläche. Es wird ferner darauf hingewiesen, dass der am meisten angewandte Trockenschrank, der bekannte einwandige Kasten aus Kupfer oder neuerdings aus Aluminium, den oben ausgesprochenen Anforderungen nicht nachkommt. Zur Erzielung einer constanten und im Trockenraum gleichmässig herrschenden Temperatur hat man Schränke mit doppelten Wandungen angewandt, die zur Aufnahme von Wasser, Kochsalzlösung, Chlorcalciumlösung 'oder von Flüssigkeiten mit noch böheren Siedepunkten eingerichtet sind. Dahin gehören die Trockenapparate nach: 1. Greff (d. Z. 1890, 326), 2. Raikow (Chemztg. 1889, 94) und 3. Victor Meyer (Ber. deutsch. G. 1885, 2999).

Mit denselben kann aber nur eine constante Temperatur erreicht werden. Zur Erzielung verschiedener constanter Temperaturen können nur eigentliche Luftbäder verwandt werden.

Dieselben sind aber meistens sehr complicirter Natur oder rechnen nicht mit dem Hauptfactor einer rationellen Trocknung, die Zuführung der Wärme nicht durch heisse Luft, sondern durch directe Leitung der 
Heizfläche zu bewirken. Dahin gehören die Apparate nach: 1. L o th ar $\mathrm{M}$ e yer (Z. anal. 1884,195$), 2$. $\mathrm{M} \mathrm{u}$ e $\mathrm{n} \mathbf{k} \mathrm{e}$ und Rohrbeck und 3. Grimshaw (Z. anal. 1892, III).

Der erste und bisher einzige Trockenschrank, der mit diesem Hauptfactor rechnet, ist der von Soxhlet (d. Z. 1891, 363). Dieser Trockenschrank gehört zu den Flüs-

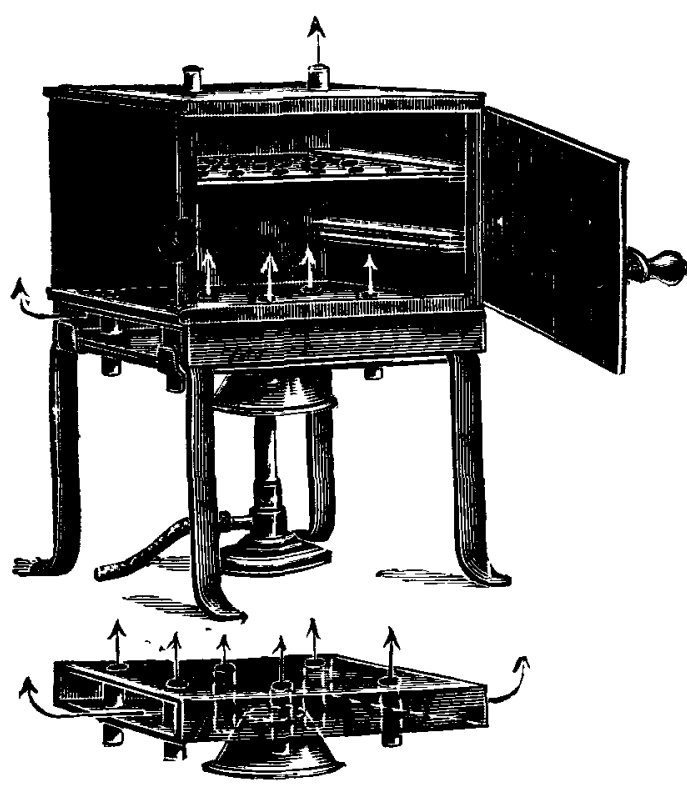

Fig. 105.

sigkeitsbädera; es können deswegen damit keine höheren Temperaturen erreicht werden. Dann besitzt derselbe zu wenig Geräumigkeit, höhere Gefässe können nicht zur Verwendung kommen. Ein Universalapparat - so schliesst der Vortrag des Herrn H. Petersen - ist demnach der Soxhlet'sche Trockenschrank noch nicht. darüber wird erst nach vielseitigem Gebrauch zu fällen sein.

Der neue Trockenkasten entspricht der 1. Anforderung: verschiedene, constante Temperaturen bei genügender Geräumigkeit zu zeigen. In der Form gleichen die neuen Luftbäder den bekannten Trockenkästen, besitzen aber gewissermaassen 2 Böden, die durch Röhren mit einander verbunden sind. Nur auf der vorderen oder Thürseite, dgl. der hinteren, sind die beiden Böden mit einander verbunden, während zu beiden Seiten ein Abzug möglich ist. Eine in der Mitte des unteren Bodens angebrachte Öffnung mit trichterförmigem Ansatz gestattet das Untersetzen eines Bunsen, dessen Flamme wie sonst den oberen Boden bespülen kann. Durch diese Anordnung entsteht ein Luftzug zunächst durch den Trichter, erwärmt sich an der Flamme und zieht, vermischt mit den heissen Verbrennungsgasen der Flamme, durch den Raum $A$. Dabei wird nicht nur der 2. Boden erwärmt, sondern auch das Röhrensystem, durch welches nunmehr ein Luftstrom aufsteigt, der in den Trockenraum mit derselben Temperatur gelangt, als der Boden zeigt und in diesem eine Luftcirculation veranlasst, welche sich durch constante Temperatur im Innern zu erkennen gibt. Die erwärmte Luft zieht durch $C$ ab. Demnach ist auch die 2. Anforderung erfüllt.

Während also die Rauchgase der Flamme nicht in das Innere gelangen können, bewirkt ein stetig im Innern aufsteigender Luftstrom eine constante Temperatur und rasche Verdampfung, unter Benutzung der Wärme durch directe Leitung von der Heizfläche. Somit ist auch die 3. Anforderung erfüllt.

Experimentelle Belege.

1. Verschiedene constante Temperatur.

\begin{tabular}{|c|c|c|c|c|}
\hline $\begin{array}{l}\text { Flamme, } \\
\text { Einbrenner }\end{array}$ & $\begin{array}{c}\text { Temperatur } \\
\text { unten }\end{array}$ & $\begin{array}{c}\text { Temperatur } \\
\text { oben }\end{array}$ & Luftbad & Bemerkungen \\
\hline $1 / 1$ & $220^{\circ}$ & $215^{0}$ & \multirow{3}{*}{$\begin{array}{l}\text { Von Kupferblech mit } \\
\text { Rohrensysteme }\end{array}$} & $\begin{array}{c}\text { Die Differenz ist der } \\
\text { verschieden erwärmten } \\
\text { Quecksilbersäule zuzu- } \\
\text { schreiben }\end{array}$ \\
\hline $1 / 2$ & $\begin{array}{l}128^{\circ} \\
104^{1} / 2^{\circ}\end{array}$ & $\begin{array}{l}127 \% \%^{0} \\
10412^{0}\end{array}$ & & $\begin{array}{l}\text { Temperatur wurde } \\
\text { mit kleinem Thermo- }\end{array}$ \\
\hline $1 / 4$ & $83^{1 / 2}{ }^{0}$ & $83^{\circ}$ & & meter gemessen \\
\hline $1 / 4$ & $91^{\circ}$ & $88^{\circ}$ & $\begin{array}{c}\text { Luftbad von Aluminium, } \\
\text { alte Construction }\end{array}$ & \\
\hline
\end{tabular}

Es steht auch der allgemeinen Verwendung der hohe Preis (120 M.) entgegen.

Angesichts dieser zu Tage liegenden Schwierigkeit in der Beschaffung eines zufriedenstellenden Trockenapparates, glaube ich durch meine neue Construction dem Universalapparat mindestens sehr nahe gekommen zu sein. Ein endgiltiges Urtheil
Temperaturen in verschiedenen Hohen bei dem Luftbad alter Construktion:

\begin{tabular}{|c|c|c|c|c|c|c|}
\hline & 1 & 2 & 3 & 1 & 2 & 3 \\
\hline Oben: & 190 & 162,5 & 158 & 212 & 200 & 20 \\
\hline Mitte: & 194 & 166,5 & 163,5 & 213 & 203,5 & \\
\hline Unten: & 211 & 195 & 192 & 230 & 217 & \\
\hline
\end{tabular}

Als weiterer Beleg für die ungleiche Temperaturvertbeilung im Aluminiumbad im 
Gegensatz zu dem veränderten Kupferluftbad neuen Systems diene folgender Versuch. 50 ce Wasser, auf 2 Porzellanschälchen gleichmässig vertheilt, gebrauchten zur vollständigen Verdampfung im Kupferkasten 2 Stunden. Die Temperatur des leer erhitzten Luftbades betrug $103^{\circ}$ und fiel während 5 Minuten auf $82^{\circ}$, um in dem Maasse, als das Wasser verdampfte, auf $100^{\circ} \mathrm{zu}$ steigen. Nach 1 Stunde 50 Minuten betrug die Temperatur $95^{\circ}$ und die Menge des verdampften Wassers $47 \mathrm{cc}$; in jedem
Porzellanschälchen waren noch $1,5 \mathrm{cc}$, zusammen 3 ce Wasser enthalten. Die Verdampfung geht also an verschiedenen Stellen der directen Heizfläche gleichmässig vor sich.

Im Gegensatz hierzu zeigte das Aluminiumbad bei der gleichen Beschickung nach 2 Stunden unter denselben Temperaturverhältnissen zwar die Verdampfung von $25 \mathrm{cc}$ Wasser desjenigen Schälchens, am Thermometer befindlich, an, während das 2. Schälchen noch 12 cc Wasser enthielt.

\begin{tabular}{|c|c|c|c|c|}
\hline Beschickung & $\begin{array}{l}\text { Dauer der } \\
\text { Verdampfung }\end{array}$ & Temperatur & Luftbad & Bemerkungen \\
\hline \multirow[t]{2}{*}{$\begin{array}{l}2 \text { Porzellanschälchen } \\
\text { à } 25 \text { ce Wasser }\end{array}$} & 2 Stunden & $\begin{array}{l}103^{\circ} \text { leer } \\
82^{\circ} \text { nach } 5 \text { M. } \\
95^{\circ}-1 \text { St. } 50 \text { M. } \\
100^{\circ}-2-\end{array}$ & $\begin{array}{l}\text { Kupfer neuer } \\
\text { Construction }\end{array}$ & $\begin{array}{l}\text { Verdampftes Wasser } \\
\text { also: } 50 \mathrm{cc}\end{array}$ \\
\hline & 2 Stunden & $\begin{array}{c}103^{\circ} \text { leer, sonst wie } \\
\text { oben }\end{array}$ & $\begin{array}{l}\text { Aluminium alter } \\
\text { Construction }\end{array}$ & $\begin{array}{l}\text { Verdampftes Wasser } \\
\text { also: } 38 \mathrm{cc} \text {, da ein } \\
\text { Schälchen noch } 12 \mathrm{cc} \\
\text { Wasser enthielt }\end{array}$ \\
\hline
\end{tabular}

Weitere Verdampfungsversuche in dem Kupferluftbad neuer Construction.

\begin{tabular}{|c|c|c|c|c|c|c|c|c|}
\hline Beschickung & 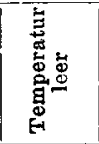 & 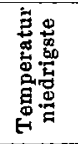 & 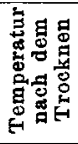 & $\stackrel{\vec{D}}{\mathbf{N}}$ & 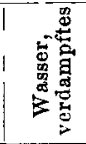 & ${ }^{1}$ Heizraum & Luftbad & \\
\hline \multirow{6}{*}{$\begin{array}{l}5 \mathrm{~g} \text { Asbest } \\
10 \mathrm{~g} \text { Wasser } \\
\text { Glasschälchen }\end{array}$} & $85^{\circ}$ & $77^{\circ}$ & $79^{\circ}$ & $30 \mathrm{M}$ & $10 \mathrm{~g}$ & directer & Kupfer & \\
\hline & $80^{\circ}$ & $70^{\circ}$ & $85^{\circ}$ & $40 \mathrm{M}$ & $10 \mathrm{~g}$ & $\begin{array}{l}\text { direct uber } \\
\text { der Flamme }\end{array}$ & \multirow[t]{2}{*}{ Aluminium } & $\begin{array}{l}\text { Schrank einmal geoffnet; geschieht dieses } \\
\text { nicht, so werden } 56 \text { Min. gebraucht }\end{array}$ \\
\hline & $124,5^{0}$ & $111^{\circ}$ & $120^{\circ}$ & $15 \mathrm{M}$ & $10 \mathrm{~g}$ & directer & & $\begin{array}{l}\text { Davon wurden } 3 \text { Versuche gemacht, die } \\
\text { alle in } 141 / 2 \text { bis } 15 \text { Min. } 10 \mathrm{~g} \text { W. ver- } \\
\text { dampften, einerlei ob die Substanz uber } \\
\text { oder neben der Flamme stand }\end{array}$ \\
\hline & $107^{\circ}$ & $96^{\circ}$ & $108^{\circ}$ & $30 \mathrm{M}$ & $10 \mathrm{~g}$ & $\begin{array}{r}\text { direct uber } \\
\text { der Flamme }\end{array}$ & Aluminium & \multirow{3}{*}{$\begin{array}{l}\text { Die Verdampfungszeit nimmt } 2 u \text {, sobald } \\
\text { die Substanz nicht der directen Heiz- } \\
\text { fläche susgesetzt wird }\end{array}$} \\
\hline & $118^{1 / 2}{ }^{\circ}$ & $111^{0}$ & $115^{0}$ & $40 \mathrm{M}$ & $10 \mathrm{~g}$ & $\begin{array}{l}\text { 1. Heizraum } \\
1 \text { Horde }\end{array}$ & \multirow{2}{*}{ Kupferbad } & \\
\hline & $118^{\circ}$ & $106^{\circ}$ & $113^{0}$ & $45 \mathrm{M}$ & $10 \mathrm{~g}$ & $\mid \begin{array}{l}\text { 2. Heizraum } \\
\text { 2. Horde }\end{array}$ & & \\
\hline
\end{tabular}

Vergleichende Verdampfungsversuche mit Wasser allein in einem Aluminiumschälchen.

\begin{tabular}{|c|c|c|c|c|c|c|c|c|}
\hline $\begin{array}{l}10 \mathrm{~g} \text { Wasser in } \\
\text { Aluminium- } \\
\text { schalchen }\end{array}$ & $124^{\circ}$ & $111^{\circ}$ & $114^{\circ}$ & $14 \mathrm{M}$. & $10 \mathrm{gW}$ & direct & Kupferbad & $\begin{array}{l}\text { Dieser Versuch wurde } 2 \text { mal wiederhoit. } \\
\text { Sobald das Wasser verdampft ist, steigt } \\
\text { die Temperatur rapid auf } 124^{\circ} \text {. }\end{array}$ \\
\hline & $126^{\circ}$ & $99^{\circ}$ & $117^{\circ}$ & $25 \mathrm{M}$ & $10 \mathrm{gW}$ & $\begin{array}{c}\text { direct } \\
\text { Substanz } \\
\text { uberFlamme }\end{array}$ & $\begin{array}{c}\text { Aluminium- } \\
\text { bad }\end{array}$ & \\
\hline & $100^{\circ}$ & $911 / 2^{0}$ & $100^{\circ}$ & $30 \mathrm{M}$. & $10 \mathrm{gW}$ & $\begin{array}{c}\text { direct } \\
\text { Substanz } \\
\text { uberFlamme }\end{array}$ & $\underset{\text { bad }}{\text { Aluminium- }}$ & \\
\hline & & & & & $10 \mathrm{~g} W$ & $\begin{array}{c}\text { direct } \\
\text { neben der } \\
\text { Flamme }\end{array}$ & $\underset{\text { bad }}{\text { Aluminium- }}$ & \\
\hline & $103^{\circ}$ & $96^{\circ}$ & $102^{\circ}$ & $14 \mathrm{M}$. & $10 \mathrm{gW}$ & direct & Kupfer & \\
\hline
\end{tabular}

Milchverdampfungen.

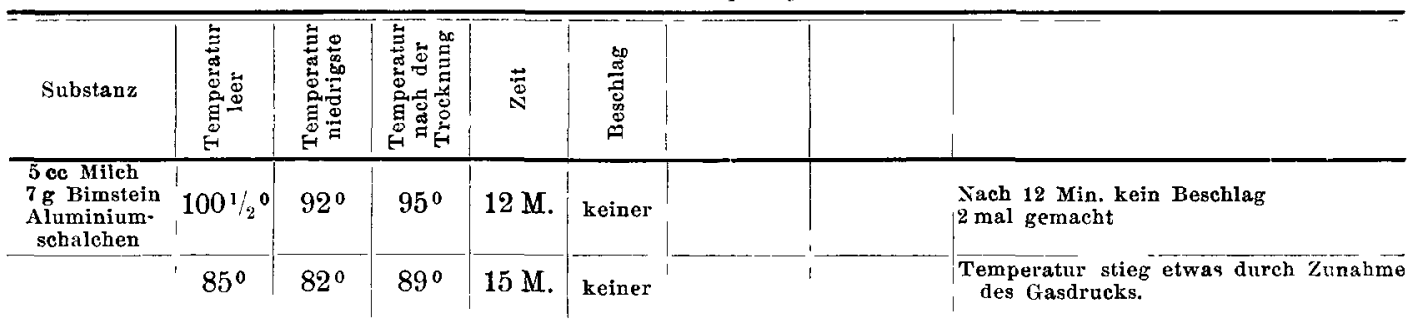


In Bezug auf die Milchbestimmungen ist zu bemerken, dass die verdampften Proben sich bei $100^{\circ}$ schwach bräunen, was aber bei 85 bis $90^{\circ}$ nicht stattfindet. Ich habe mich lediglich nach dem Beschlag gerichtet, der bei Wasserverdampfungen mit seltener Schärfe den Endpunkt der Verdampfung abgibt. Soxhlet gibt für die gleiche Beschickung 16 Minuten an.

Die Tabellen zeigen, wie die Verdampfung im Kupferluftbad bedeutend schneller stattfindet, was umsomehr hervortritt, wenn die zu verdampfenden Proben nicht über die Flamme gestellt werden.

Die neuen Trockenschränke sind gesetzlich geschützt und werden von der Fabrik chemischer Apparate, Max Kaehler \& Martini, Berlin W., hergestellt.

\section{Hüttenwesen.}

Schnelle und sichere Methode zur Bestimmung von Silber und Gold in Metallen, Erzen u. s. w. von C. Whitehead (Proc. Frankl. Inst. 1892). Je nach der Reichhaltigkeit werden z. B. 10 bis $20 \mathrm{~g}$ Robkupfer in einem $500 \mathrm{cc}$-Becherglas eingewogen, durch allmäbliches Zusetzen ron Salpetersäure gelöst, die rothen Dämpfe durch Erhitzen vertrieben, mit Wasser verdünnt und $50 \mathrm{~g}$ Bleiacetat zugefügt. Nach der Aufösung wird 1 cc verd. Schwefelsäure zugefügt, nach Absetzen des Niederschlages in einen Literkolben filtrirt und bis zur Marke aufgefüllt. Das Filter, Bleisulfat nebst dem Golde und vielleicht etwas Silber baltend, wird eingeäschert, mit Probirblei behandelt und cupellirt. Das Gold wird gewogen, wie gewöhnlich mit Silber quartirt und das etwa dabei gefundene Silber in Rechnung gestellt. Das erste Filtrat wird in zwei gleiche Theile getheilt und die Doppelprobe so lange mit einer concentrirten Bromnatriumlösung versetzt, bis kein Niederschlag mehr entsteht. Der Niederschlag, der sich schnell absetzt, wird abfiltrirt und mit kaltem Wasser gut ausgewaschen. Etwa in Lösung gegangenes Bromblei ist ohne Bedeutung. Der getrocknete Niederschlag, der sich leicht vom Filter entfernen lässt, wird mit einer dreifachen Menge Soda und irgend einem reducirenden Mittel, wie Mebl gemischt, in einen kleinen Tiegel gebracht, mit Boraxglas gedeckt und im Muffel eingeschmolzen. Der Regulus wird etwa $2 \mathrm{~g}$ wiegen und, wenn gut ausgewaschen, frei von Kupfer und sonstigen Verunreinigungen sein. Derselbe wird nun bei niedriger Temperatur abgetrieben. Die beigegebenen Zahlen zeigen gute Übereinstimmung.

Jodometrische Bestimmung von Nitraten von F. A. Gooch und H. W. Grüner (Journ. Anal. 1892 S. 401). Der Versuch, die Nitrate in derselben Weise wie die Chlorate (d. Z. 1892 S.90) zu bestimmen, misslang, da mit der schwachen Säure die Nitrate sich nicht vollkommen zersetzten. Auf dem folgenden Wege gelang aber eine völlige Zersetzung: Sättigt man conc. Salzsäure mit Manganchlorür und bringt dieses mit einem Nitrat zusammen, so findet bei gewöhnlicher Temperatur nur eine geringe Einwirkung statt. Beim Erwärmen aber bilden sich höhere Chloride des Mangans unter Freilassen von Stickoxyd. Bei weiterem Erhitzen wird das Manganchlorür regenerirt und das überschüssige Chlor entweicht. Die ursprüngliche grüne Farbe des Reagens wird hierbei anfangs immer dunkler bis zu schwarz, um beim Ende des Erhitzens ihre ursprüngliche Farbe wieder zu erlangen. Um eine vollständige Zersetzung des Nitrates zu erzielen, muss mehr als das Dreifache der Nitratlösung an Reagens vorhanden sein. Denn mit genügend Wasser ist man im Stande, die ganze Reaction aufzuheben. Wie Nitrate wirken auch Chlorate und sonstige Verbindungen, die mit Salzsäure Chlor entwickeln. Sind solche aber abwesend, so kann die Reaction auch zu qualitativem Nachweis von Nitraten dienen.

Das Nitrat sammt Manganlösung wird in irgend einen für Chlordestillation benutzten Apparat gebracht. Als Retorte wird eine liegende Pipette gebraucht. Dieselbe ist mit einem Stickstoff kugelapparat verschmolzen, dessen Spitze ausgezogen wird, damit sie in das Verbindungsrobr zu dem darauf folgenden Absorptionskolben geschoben werden kann. In das Kugelrohr, das während der Arbeit abgekühlt wird, kommt eine Lösung von $3 \mathrm{~g}$ Jodkalium, in den Kolben eine von $1 \mathrm{~g}$ und in das Absperrrohr nur einige Zehntelg. Nachdem durch gelindes Saugen die Nitrat- und Manganlösung in die Retorte gebracht ist, wird sofort die Luft aus dem Apparat durch Kohlensäure verdrängt. Hierauf wird die Retorte erhitzt und der grösste Theil der Flüssigkeit überdestillirt. Nach beendeter Destillation wird der Inhalt der Retorte sammt Empfänger (die Manganflüssigkeit ist ohne irgend welchen Einfluss) in ein Becherglas gespült und das ausgeschiedene Jod titrirt. Die Titrirung muss sofort nach dem Entleeren stattfinden. Die beigegebenen Zahlen zeigen schöne Übereinstimmung. 